\title{
Relações entre a Compreensão de Leitura, Resolução de Problemas de Raciocínio Quantitativo e Funções Executivas
}

\section{Relations Between Reading Comprehension, Quantitative Reasoning Problem Solving and Executive Functions}

\author{
(iD)
iD
(iD)
iD
iD \\ Évelin Fulginiti de Assis ${ }^{1}$ \\ Camila Peres Nogues ${ }^{1}$ \\ Luciana Vellinho Corso ${ }^{1}$ \\ Beatriz Vargas Dorneles ${ }^{1}$ \\ Helena Vellinho Corso ${ }^{2}$ \\ 'Universidade Federal do Rio Grande do Sul (UFRGS), Programa de Pós-Graduação em Educação, Porto Alegre, \\ RS, Brasil. Autora Correspondente: evelin.assis@ufrgs.br \\ ${ }^{2}$ Universidade Federal do Rio Grande do Sul (UFRGS), Faculdade de Educação, Departamento de Estudos \\ Especializados, Porto Alegre, RS, Brasil.
}

Resumo: Esta pesquisa teve como objetivo verificar as relações entre o desempenho em tarefas de resolução de problemas de raciocínio quantitativo, de leitura e de funções executivas em alunos de quinto ano do Ensino Fundamental. Além disso, foi verificada a presença do efeito de consistência, relacionado à concordância entre a linguagem do enunciado e a operação a ser utilizada nos problemas matemáticos. Os resultados evidenciaram relações (correlação de Spearman) entre as tarefas de: resolução de problemas, reconhecimento de palavras e compreensão de leitura, apontando para o importante papel da realização de inferências. Também foram encontradas correlações significativas entre as medidas de funções executivas, de compreensão de leitura e de resolução de problemas, com destaque para a memória de trabalho. O efeito de consistência foi encontrado (comparação de Wilcoxon), demonstrando que problemas cuja linguagem é consistente com a operação envolvida são mais fáceis de resolver do que os de linguagem inconsistente.

Palavras-chave: Ensino fundamental; Resolução de problemas; Raciocínio lógico matemático; Leitura compreensiva; Consistência cognitiva.

Abstract: This research aimed to verify the relations between the performance of 5 th graders in tasks of quantitative reasoning problem solving, reading and executive functions. Furthermore, the presence of the consistency effect was verified, which relates to the agreement between the wording of the mathematical problem and the operation required to solve it. The results evidenced relations (Spearman correlation) between the following tasks: problem solving, word recognition and reading comprehension, thus indicating the important role of inferences. Significant correlations were also demonstrated between measures of executive functions, reading comprehension and problem solving, with an emphasis on working memory. The consistency effect was found (Wilcoxon comparison), showing that problems in which language is consistent with the operation involved are easier to solve than problems with inconsistent language.

Keywords: Elementary school; Problem solving; Mathematical logical reasoning; Comprehensive reading; Cognitive consistency.

Recebido em: 06/07/2020

Aprovado em: 05/10/2020 


\section{Introdução}

As habilidades de leitura e a matemática envolvem diversos aspectos cognitivos, de domínio específico e de domínio geral, e a compreensão das relações entre tais facetas é fundamental para o favorecimento dos processos de ensino e aprendizagem. Este artigo descreve como a resolução de problemas de raciocínio quantitativo e a compreensão de leitura estão relacionadas entre si e com as funções executivas.

O desempenho acadêmico dos alunos brasileiros nas avaliações de larga escala revela uma realidade educacional preocupante. Os resultados do Programa Internacional de Avaliação de Estudantes (PISA) mostram que, na área da leitura, apenas $50 \%$ dos estudantes brasileiros (contra $77 \%$ da média da Organização para a Cooperação e Desenvolvimento Econômico, OCDE) é capaz de identificar a ideia principal de um texto de tamanho moderado, encontrar informações baseadas em critérios explícitos, às vezes complexos, e refletir sobre a forma e o propósito de textos quando diretamente solicitados a fazê-lo (OECD, 2019). Já na área da matemática, apenas $32 \%$ dos alunos (a média da OCDE foi de 76\%) consegue reconhecer e interpretar, sem instruções diretas, como uma situação simples pode ser representada matematicamente (OECD, 2019). Esses resultados evidenciam a necessidade de uma discussão mais profunda sobre os elementos envolvidos no ensino e na aprendizagem de tais áreas. Um relevante passo nesse sentido é compreender que, embora as aprendizagens da leitura e da matemática possam caracterizar processos específicos, existem relações entre elas, e as especificidades de uma área acabam influenciando a outra (CORSO; DORNELES, 2015). Também há o importante papel das competências de base, especificamente as funções executivas (FE). De forma especial, a resolução de problemas com enunciado evidencia o papel da linguagem e da compreensão de leitura no desempenho matemático, visto que supõe a inferência do cálculo ou raciocínio a ser feito a partir de informação linguística (FLETCHER et al., 2009). O presente estudo examina as relações entre o desempenho em tarefas de resolução de problemas matemáticos, tarefas de leitura e de funções executivas (FE) de 57 alunos de $5^{\circ}$ ano do Ensino Fundamental.

\section{Aprendizagem da Matemática e Raciocínio Quantitativo}

O ensino e a aprendizagem da matemática pressupõem a compreensão de um conjunto de condições individuais, ambientais e escolares que atuam de maneira integrada (CORSO; ASSIS, 2018). A matemática possui uma estrutura hierárquica, de modo que as habilidades previamente construídas são pré-requisitos para a construção de novas habilidades e, nesse sentido, crianças com dificuldades em habilidades iniciais, por exemplo, não terão uma base sólida para a construção de novas habilidades, o que pode acarretar problemas posteriores (CORSO; ASSIS, 2018). Tais dificuldades podem se manifestar por meio de déficits em habilidades acadêmicas, como cálculo e resolução de problemas, mas a matemática é uma área ampla que vai além de cálculos aritméticos, envolvendo diversas competências numéricas. Essas, por sua vez, são resultado de um processo de construção da criança, que se inicia com experiências informais e vai tornando-se mais complexo na medida em que as experiências de ensino formal ocorrem (CORSO; DORNELES, 2013). As competências de base, por sua vez, correspondem aos processos cognitivos que dão suporte à 
execução de diversas ações. Dorneles, Lima e Nogues (2018) destacam que o raciocínio lógico se apresenta como a base para a compreensão das relações entre quantidades, usadas tanto para ensinar quanto para aprender matemática, sendo a capacidade de organização do pensamento que ajuda a resolver problemas e chegar a uma conclusão.

Nunes et al. (2012) evidenciaram uma for te ligação entre o raciocínio e o desempenho matemático das crianças, indicando que um maior investimento no desenvolvimento desse raciocínio pode produzir resultados melhores em desempenho matemático: ainda que o raciocínio matemático e a aritmética tenham sido correlacionados moderadamente, suas contribuições independentes na predição da performance matemática demonstram que estes devem ser tratados como construtos distintos. Nesse sentido, Nunes et al. (2016) ainda explicam: enquanto a aritmética se baseia nas relações entre os números, o raciocínio quantitativo é baseado nas relações entre as quantidades, dividindo-se em dois tipos: raciocínio aditivo e raciocínio multiplicativo. O primeiro raciocínio envolve relações parte-todo e o segundo, por sua vez, envolve relações de correspondência um para muitos ou razão. As situações parte-todo podem ser classificadas em três tipos: transformação, comparação e composição. As situações de correspondência um para muitos ou razão podem ser classificadas em relação direta, relação inversa, produto de medidas e proporcionalidade.

\section{Influências Linguísticas no Processamento Matemático}

A resolução de problemas se presta especialmente para o exame das influências linguísticas no processamento matemático. O que caracteriza os problemas matemáticos com enunciado verbal (word problem) é que as informações importantes são apresentadas por meio de uma pequena narrativa, em vez de uma notação matemática (VERSCHAFFEL; GREER; DE CORTE, 2000). Neste tipo de problema, acresce-se ao fator numérico o aspecto linguístico. Daroczy et al. (2017) apresentam um modelo de processamento de resolução de problemas com enunciado, enfocando os fatores linguísticos e numéricos que contribuem para dificuldades na sua resolução (Figura 1):

Figura 1 - Modelo de processamento de resolução de problemas com enunciado

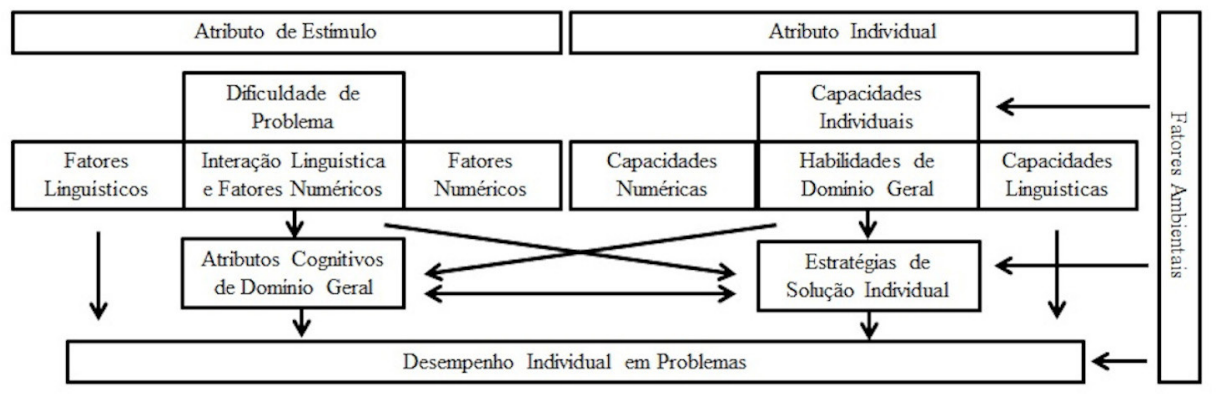

Fonte: Daroczy et al. (2017, tradução nossa).

Daroczy et al. (2017) ressaltam que os atributos de estímulo e os individuais podem influenciar, de forma direta, o desempenho em problemas e também por meio de duas variáveis mediadoras: os atributos de domínio geral e as estratégias de solução individual. A complexidade numérica ou linguística pode aumentar a carga cognitiva e esseaumento pode ser superaditivo, especialmente quando a carga ultrapassa a capacidade individual. Além 
disso, indivíduos com diferentes habilidades linguísticas ou numéricas poderão enfrentar uma carga cognitiva diferente para um mesmo problema: por exemplo, um sujeito com tais habilidades maiores enfrentará uma carga menor do que aquele com menores habilidades.

Já as estratégias específicas de solução podem facilitar a resolução de problemas, uma vez que, se determinada estratégia pode ser aplicada para um problema em particular, porque o tipo de problema permite e porque o sujeito conhece a estratégia, a solução é atingida mais facilmente. Os fatores ambientais, como o ensino, influenciam tanto as capacidades individuais quanto as estratégias de solução e o próprio desempenho em problemas. O modelo apresentado pelos autores busca facilitar a compreensão dos aspectos envolvidos na resolução de problemas, evidenciando que diferentes fatores atuam em conjunto e podem influenciar uns aos outros durante o processo de solução (DAROCZY et al., 2017).

Um fator relacionado diretamente à dificuldade na resolução de problemas diz respeito às palavras utilizadas em seu enunciado, que acabam atuando como pistas que levam à solução, como $e$, mais que, menos que, cada um, dentre outras. De acordo com Dowker e Nuerk (2017), o significado das palavras também influencia o processamento numérico ou aritmético. Os autores destacam que os problemas são mais fáceis de resolver quando a operação necessária é consistente com a palavra utilizada no problema (por exemplo, a palavra usada é mais e a operação é de adição) e que tanto adultos quanto crianças apresentam um desempenho pior quando não há esta consistência. Este é um exemplo de influência linguística a nível semântico, podendo-se identificar outros níveis linguísticos que também influenciam o processamento numérico e aritmético: conceitual, sintático, lexical, ortográfico viso espacial e fonológico (DOWKER; NUERK, 2017).

\section{Aprendizagem da Leitura}

A aprendizagem da leitura é um processo longo, que se inicia pela habilidade de reconhecer palavras, avança com uma decodificação rápida e precisa, e culmina com a capacidade de compreender textos longos. A compreensão de leitura é uma atividade complexa que, assim como a matemática, supõe aspectos específicos e gerais. Os processos específicos do texto (fluência na decodificação e estrutura do texto) interagem com processos socioemocionais e cognitivos (como as funções executivas) e com os processos linguísticos (que incluem o sistema léxico e o sistema semântico). Esses três fatores, aportados pelo aluno, interagem entre si, tendo um efeito impulsionador recíproco e sofrendo o efeito da instrução (na escola e em casa) (CONNOR, 2016).

O leitor de um texto processa inicialmente as palavras individuais, acessando suas representações fonológicas, ortográficas e semânticas, que são conectadas para que haja a compreensão da frase. De forma similar, para compreender o texto como um todo, o leitor precisa processar e conectar unidades de ideias individuais, resultando na construção de uma representação mental coerente do texto na memória do leitor (KENDEOU et al., 2014). A representação mental que resulta da leitura não é uma cópia do texto, mas, pelo contrário, corresponde a uma síntese construída de forma ativa a partir de relações estabelecidas entre a informação do texto e o conhecimento prévio do leitor (CORSO; SPERB; SALLES, 2013). Processos executivos garantem a habilidade de organizar e refletir sobre a informação nos limites da memória de trabalho (KENDEOU et al., 2014), 
de onde a pesquisa empírica vem comprovando o papel central das funções executivas na habilidade de compreender textos (CORSO et al., 2016).

\section{Funções Executivas}

As funções executivas desempenham papel central tanto na aprendizagem da matemática como no desenvolvimento da compreensão de leitura. Uma revisão sistemática incluiu 66 estudos que exploraram a associação entre FE e o rendimento em matemática e leitura, e verificou, por meio de técnicas meta-analíticas, que existem associações moderadas incondicionais entre $\mathrm{FE}$ e rendimento nas duas habilidades acadêmicas, que não diferem de acordo com o componente de FE considerado (memória de trabalho, resposta inibitória, controle atencional, flexibilidade cognitiva), ou idade, ou o tipo de medida usado (JACOB; PARKINSON, 2015).

Cabe uma consideração teórica específica sobre as FE - já que aparecem como um correlato cognitivo comum à aprendizagem da matemática e da leitura compreensiva -, com base na qual justificamos o modo de operacionalização do conceito neste trabalho. Diferentes definições para o conceito de funções executivas, assim como para seus possíveis subcomponentes, podem ser encontradas na literatura. A variedade de processos e funções que aparecem associados ao termo pode gerar alguma dificuldade conceitual, bem como confusão quanto à operacionalização do conceito na pesquisa empírica. Há, de todo modo, consensos importantes: o funcionamento executivo é essencial para o comportamento humano, pois gerencia todos os recursos cognitivos e comportamentais para planejar e regular as ações com propósitos, seja no terreno cognitivo, como no socioemocional (CORSO; SPERB; SALLES, 2013).

O construto é complexo e multifacetado. Diamond (2013) descreve três funções executivas nucleares - o controle inibitório (foca e mantém a atenção, minimizando a interferência de estímulos alheios à tarefa), a memória de trabalho (mantém e processa a informação envolvida na atividade em curso), e a flexibilidade cognitiva (permite a mudança de foco diante das mudanças do ambiente, possibilitando soluções de problemas e respostas criativas). A autora destaca que, com base nessas três principais funções executivas, são construídas as funções executivas de ordem superior, como o raciocínio, resolução de problemas e planejamento. O raciocínio e a resolução de problemas podem ser compreendidos como sinônimos de inteligência fluída, que envolve a capacidade de entender as relações abstratas subjacentes a analogias (DIAMOND, 2013). Compreende-se, assim, o envolvimento das funções executivas na resolução de problemas de raciocínio quantitativo, utilizados neste artigo, como um aspecto da aprendizagem da matemática. Um fator muito importante ao se considerar as funções executivas e seu papel na aprendizagem da leitura e da matemática é o fato de que elas se desenvolvem a partir da interação com o ambiente, isto é, a experiência tem um papel central nas capacidades executivas. Tanto é assim, que inúmeras pesquisas de intervenção já atestaram a eficiência do treinamento executivo (CORSO et al., 2018).

\section{Relações entre Desempenho Matemático, Desempenho em Leitura e Funções Executivas}

A literatura apresenta evidências que possibilitam a reflexão e a análise dos aspectos comuns e/ou relacionados entre o desempenho matemático, desempenho em 
leitura e funções executivas. Martin et al. (2014) demonstraram que medidas de domínio geral como memória de trabalho e consciência fonológica, juntamente com medidas de domínio específico, como contagem e número simbólico, avaliadas na Educação Infantil, foram preditoras de fluência em cálculo, cálculo e resolução de problemas no $1^{\circ}$ ano do Ensino Fundamental. Já no estudo de Hassinger-Das et al. (2014), o funcionamento executivo foi mais importante para a aprendizagem conceitual da matemática, especialmente problemas matemáticos orais, do que para a aprendizagem procedimental, sendo que a medida de problemas orais foi correlacionada com medidas de leitura, senso numérico e funções executivas. Estudos que investigam os perfis cognitivos de alunos com dificuldades em leitura, em matemática e dificuldades em leitura e matemática também fornecem boas evidências para análise. Corso e Dorneles (2015) identificaram que alunos com dificuldades em leitura apresentam prejuízos na consciência fonológica e velocidade de processamento, enquanto alunos com dificuldades em matemática apresentam prejuízos na memória de relatos e estratégias imaturas de contagem, assim como aqueles com dificuldades nas duas áreas. Este grupo demonstrou ser o mais prejudicado, evidenciando dificuldades em todas as tarefas cognitivas (processamento fonológico e memória de trabalho). Tal resultado também foi evidenciado por Cirino et al. (2015), cujo estudo apontou que o grupo de alunos com dificuldades em leitura e matemática obteve desempenho inferior aos grupos com dificuldades apenas em leitura ou apenas em matemática, tanto nas medidas cognitivas (velocidade de processamento, memória de trabalho, consciência fonológica e subtestes de vocabulário e raciocínio) quanto nas medidas matemáticas (competências numéricas fundamentais, fatos básicos, problemas orais e escritos): o grupo com dificuldades nas duas áreas de conhecimento apresentou fraquezas adicionais em numeração e problemas matemáticos, com um perfil mais prejudicado do que o grupo com dificuldades em matemática nas tarefas da área.

Destaca-se, em especial, o trabalho de Jacob e Parkinson (2015), que consiste em uma revisão de trabalhos de intervenção escolar para melhorar o desempenho acadêmico em leitura e/ou matemática. Conforme mencionado anteriormente, foram analisados 66 estudos, com 43 explorando a relação entre desempenho e funções executivas e 23 investigando a associação preditiva entre estas variáveis. Os resultados evidenciaram uma relação direta do desempenho com funções executivas, indicando que quanto melhor a capacidade de funções executivas do estudante, melhor será o seu desempenho tanto em leitura quanto em matemática. A análise dos subcomponentes das funções executivas, separadamente, evidenciou que a memória de trabalho e o shifting atencional aparentam ser mais fortemente correlacionados com desempenho em leitura do que o controle atencional ou a resposta à inibição. Já em relação à matemática, a associação é mais forte para controle atencional e inibição.

É importante destacar que os estudos que envolveram resolução de problemas empregaram tarefas em que o problema foi lido oralmente pelo aplicador. A apresentação de problemas matemáticos de forma oral ou escrita implica diferentes estratégias. Alguns autores (CIRINO et al., 2015; HASSINGER-DAS et al., 2014) utilizam a apresentação oral dos problemas justamente para não envolver habilidades de leitura e/ou compreensão de leitura, tornando a resolução do problema menos complicada para os alunos.

Em suma, a literatura indica: (a) relações entre linguagem e leitura de um lado, e desempenho matemático de outro; e, (b) funções executivas como um correlato 
cognitivo comum para o aprendizado da matemática e da leitura compreensiva. Nesse sentido, o objetivo deste estudo é verificar as relações entre os desempenhos em tarefas de resolução de problemas de raciocínio quantitativo, tarefas de leitura (reconhecimento de palavras, fluência e compreensão) e tarefas de funções executivas em 57 alunos do $5^{\circ}$ ano do Ensino Fundamental. Adicionalmente, temos como objetivo verificar a presença do efeito de consistência nos problemas matemáticos (DAROCZY et al., 2017; DOWKER; NUERK, 2017). Como hipótese, espera-se encontrar relações significativas entre as tarefas de leitura, de matemática (raciocínio quantitativo) e funções executivas. Espera-se encontrar, também, o referido efeito de consistência, havendo mais acertos nos problemas consistentes do que nos inconsistentes.

\section{Método}

Esta é uma pesquisa transversal, que se caracteriza como uma investigação com coleta de dados realizada em determinado período de tempo (de março a abril de 2019), de caráter quantitativo e que faz parte do projeto de pesquisa inscrito na Plataforma Brasil e aprovado pelo Comitê de Ética em Pesquisa da Universidade Federal do Rio Grande do Sul (UFRGS) sob o número 70460017.3.0000.5347.

\section{Participantes}

Participaram da pesquisa 57 alunos (54,8\% meninos), com idades entre 9 e 12 anos $(M=10,3 ; D P=0,63)$ de duas escolas estaduais de Porto Alegre. Foi obtida a autorização dos pais/responsáveis por meio da assinatura do Termo de Consentimento Livre e Esclarecido. Os critérios de inclusão foram ausência de histórico de doenças neurológicas e/ou psiquiátricas, bem como de dificuldades visuais ou auditivas não corrigidas (relatadas pelos responsáveis), desempenho igual ou superior ao percentil 25 no teste Matrizes Progressivas Coloridas de Raven, escala especial (ANGELINI et al., 1999), e português como língua materna. Juntamente com o teste de Raven, foi utilizado, para garantia dos critérios de inclusão, o questionário socioeconômico, adaptado da Associação Brasileira de Empresas de Pesquisa (2009) e de condições de saúde e escolarização (preenchidos pelos pais/ responsáveis).

\section{Procedimentos e Instrumentos}

As crianças foram avaliadas no ambiente escolar em ocasiões previamente combinadas com a escola. Para a avaliação coletiva do quociente de inteligência e a avaliação individual das funções executivas foram aplicados, por uma psicóloga, os respectivos instrumentos:

- Matrizes Progressivas Coloridas de Raven (ANGELINI et al., 1999): teste para aferir o quociente de inteligência do sujeito;

- NEUPSILIN-Inf (SALLES et al., 2016): foram usadas as tarefas da bateria que correspondem às funções de atenção, memória de trabalho e funções executivas: cancelamento de figuras (atenção visual); repetição de sequência de dígitos na ordem direta (atenção auditiva); repetição de sequência de dígitos na ordem indireta (componentes fonológico e executivo central da memória de trabalho); 
repetição de sequência de blocos na ordem indireta (componentes viso espacial e executivo central da memória de trabalho); fluência verbal (ortográfica e semântica); tarefa go/no go.

A avaliação da leitura e da compreensão de leitura foi realizada individualmente por membros da equipe de pesquisa:

- Avaliação da leitura de palavras isoladas (LPI) (SALLES; PICCOLO; MINÁ, 2017): avalia a precisão na leitura oral de palavras isoladas. A tarefa é constituída por estímulos (palavras regulares, irregulares e pseudopalavras) selecionados conforme regularidade, extensão, frequência e lexicalidade, permitindo avaliar a funcionalidade da leitura, segundo os modelos de dupla-rota;

- Avaliação da fluência de leitura textual (AFLeT) (BASSO et al., 2018): envolve a leitura oral do texto integral e resposta ao questionário, abrangendo diferentes aspectos da fluência (velocidade, precisão e prosódia), além da compreensão leitora;

- Avaliação da Compreensão de Leitura Textual (COMTEXT) (CORSO et al., 2017): composto por duas tarefas que avaliam de forma qualitativa e quantitativa a compreensão de leitura de um texto narrativo: reconto (recordação livre) e respostas a questões (literais e inferenciais) de múltipla escolha, a partir da leitura silenciosa da história.

O raciocínio quantitativo foi avaliado coletivamente:

- Problemas de Raciocínio Quantitativo (NUNES, 2009): avaliação do raciocínio quantitativo (aditivo e multiplicativo) por meio da resolução, pelos alunos, de 18 problemas matemáticos (nove de raciocínio aditivo e nove de raciocínio multiplicativo) lidos oralmente pela aplicadora.

Para a análise do efeito de consistência (DAROCZY et al., 2017; DOWKER; NUERK, 2017), os problemas foram classificados pelas autoras em consistentes e inconsistentes. Os critérios utilizados foram: a análise do enunciado do problema, sem considerar a imagem, e o enfoque nas expressões relacionadas ao raciocínio solicitado (por exemplo: quando a expressão a mais estivesse empregada em um problema cujo raciocínio envolvesse a ideia de adição, o problema foi considerado consistente; se o mesmo termo estivesse envolvido em um problema de subtração, a questão foi classificada como inconsistente). No processo de classificação, houve concordância unânime das autoras em oito problemas, sendo quatro consistentes e quatro inconsistentes. Cinco problemas foram classificados com a concordância da maioria das autoras, sendo um consistente e quatro inconsistentes. Do restante, cinco foram retirados da análise por serem considerados neutros (isto é, a linguagem considerada como potencialmente não influente para a resolução). Dessa forma, foi analisado o efeito de consistência em 13 problemas (oito inconsistentes e cinco consistentes).

\section{Análise de Dados}

Para investigar se há relações entre as tarefas de compreensão de leitura, de raciocínio quantitativo (aditivo e multiplicativo) e de funções executivas, foi realizada uma análise de correlação de Spearman, visto que os dados apresentaram uma distribuição não paramétrica (teste de normalidade Shapiro-Wilk). A análise de correlação visa verificar se determinadas variáveis apresentam dependência, isto é, algum tipo de 
relação estatisticamente comprovada (BUSSAB; MORETTIN, 2010), podendo indicar uma relação positiva, ou seja, quando o aluno vai bem em uma variável, também vai bem na outra, ou negativa, isto é, quando o estudante vai bem em uma variável, o contrário ocorre na outra. A relação medida por este teste é expressa por meio do índice $r$, que indica valores entre -1 e 1, portanto quanto mais próximo de -1 ou 1 , mais forte será a correlação entre as variáveis consideradas. Além disso, esse valor tem sua probabilidade de significância avaliada e expressa por meio do $p$-valor: quando o r apresentar $p$-valor menor que 0,05, verifica-se que a correlação é significativa. Também foi conduzida uma análise de consistência dos problemas de raciocínio quantitativo, considerando a classificação mencionada anteriormente. Porém, como o número de questões em cada tipo de problema foi diferente, optou-se por considerar a proporção do total de acertos para igualar a quantidade de problemas consistentes e inconsistentes. Então, foi realizado teste de comparação não paramétrico de Wilcoxon, com a intenção de verificar possíveis diferenças entre o desempenho dos estudantes de acordo com a consistência do enunciado do problema.

\section{Resultados e Discussão}

As análises descritivas dos desempenhos dos alunos podem ser verificadas na Tabela 1, na qual são apresentadas as médias, desvios padrão, mínimos e máximos de acertos em cada uma das tarefas. A seguir, também são descritos e analisados os resultados de interesse.

\section{Relações entre tarefas de leitura e problemas de raciocínio quantitativo}

Quando se tomam as três variáveis relacionadas à matemática - total de acertos no raciocínio quantitativo (RQ), que envolve o total de acertos de questões de raciocínio aditivo ( $R A$ ) e de multiplicativo ( $R M$ ) - verificam-se correlações positivas e significativas com variáveis relacionadas à compreensão de leitura, especificamente com aquelas relacionadas à capacidade de fazer inferências: total de inferências no reconto ( $R Q: r=0,473, p<0,01$; $R A: r=0,341, p<0,01$; $R M: r=0,398, p<0,01$ ) e questões inferenciais $(r(R Q)=0,357, p<0,01 ; r(R A)=0,507, p<0,01 ; r(R M)=0,278, p<0,05)$, presentes nos instrumentos Anele 2 e 5, respectivamente. Esse resultado aponta que a resolução de problemas matemáticos lidos oralmente tem relação com a compreensão de leitura no que diz respeito à realização de inferências. Em outras palavras, no grupo avaliado, quanto maior a capacidade dos alunos em realizar inferências, melhor foi o seu desempenho no raciocínio quantitativo. A inferência é uma relação feita mentalmente, no sentido de preencher as informações deixadas implícitas no texto (CORSO et al., 2017): a capacidade de fazer inferências é central na compreensão de leitura e é, em si, um raciocínio, de modo que este achado não surpreende. Ao mesmo tempo, a resolução de problemas supõe justamente a inferência acerca da operação a ser feita, operação esta que não está explícita no enunciado verbal.

Por outro lado, ao se tomar apenas o escore total do desempenho matemático, isto é, ao considerar somente o raciocínio quantitativo, amplia-se o conjunto de variáveis de leitura que apresentam relações significativas com a tarefa de matemática, de forma semelhante aos resultados de Hassinger-Das et al. (2014) e Cirino et al. (2015). 
Encontram-se correlações com as variáveis ligadas ao reconhecimento de palavras (Anele 1): leitura de palavras reais $(r=0,270, p<0,05)$ e leitura de palavras reais irregulares $(r=0,284$, $\mathrm{p}<0,05$ ); com as variáveis ligadas à compreensão de texto (Anele 2): total de acertos no questionário $(r=0,288, p<0,05)$, total de acertos do questionário literal $(r=0,339, p<0,01)$, total de cláusulas recontadas $(r=0,356, p<0,01)$, porcentagem da cadeia principal da história presente no reconto $(r=0,332, p<0,05)$ e total de reconstruções no reconto $(r=$ $-0,265, p<0,05)$; e com as variáveis ligadas à fluência na leitura de texto e compreensão (Anele 5): total de acertos no questionário $(r=0,268, p<0,05)$ e tempo total de leitura ( $r=$ $-0,308, p<0,05)$. A partir de tais dados, destaca-se que o desempenho geral em resolução de problemas de raciocínio quantitativo está associado ao melhor desempenho em leitura e compreensão de leitura, isto é, pode-se dizer que os desempenhos nas duas áreas são dependentes. Assim, nesta amostra, a habilidade de raciocinar sobre as relações entre as quantidades em tarefas matemáticas variou junto com a fluência de leitura, com o reconhecimento de palavras e com a compreensão do texto lido.

Tabela 1 - Desempenho dos participantes em cada tarefa avaliada

\begin{tabular}{|c|c|c|c|c|}
\hline & Média & $\begin{array}{l}\text { Desvio } \\
\text { padrão }\end{array}$ & Mínimo & Máximo \\
\hline \multicolumn{5}{|l|}{ Anele 1} \\
\hline LPI Total de acertos & 54,39 & 3,78 & 45 & 59 \\
\hline LPI Palavras Reais & 37,96 & 2,11 & 32 & 40 \\
\hline LPI Palavras Reais Regulares & 19,47 & 0,80 & 17 & 20 \\
\hline LPI Palavras Reais Irregulares & 18,54 & 1,51 & 15 & 20 \\
\hline LPI Pseudopalavras & 16,47 & 2,31 & 11 & 20 \\
\hline \multicolumn{5}{|l|}{ Anele 2} \\
\hline Total de acertos questionário & 6,44 & 2,29 & 1 & 10 \\
\hline Total de acertos literais & 3,89 & 1,19 & 1 & 5 \\
\hline Total de acertos inferenciais & 2,54 & 1,48 & 0 & 5 \\
\hline Total de cláusulas & 10,02 & 5,32 & 0 & 23 \\
\hline Porcentagem cadeia principal & 44,64 & 23,29 & 0,00 & 87,50 \\
\hline Total de Inferências & 1,04 & 2,27 & 0 & 16 \\
\hline Total de Reconstruções & 2,09 & 1,88 & 0 & 7 \\
\hline \multicolumn{5}{|l|}{ Anele 5} \\
\hline Total de acertos & 8,38 & 1,49 & 3 & 10 \\
\hline Questões literais & 4,50 & 0,74 & 2 & 5 \\
\hline Questões inferenciais & 3,88 & 1,11 & 1 & 5 \\
\hline Tempo de leitura (minutos) & 2,19 & 0,61 & 1,43 & 4,72 \\
\hline \multicolumn{5}{|l|}{ Funções Executivas } \\
\hline Atenção & 53,88 & 4,07 & 43 & 59 \\
\hline Memória de Trabalho & 52,79 & 9,16 & 37 & 73 \\
\hline Fluência Verbal & 20,47 & 6,75 & 8 & 38 \\
\hline GO/NO-GO & 56,60 & 4,65 & 30 & 60 \\
\hline \multicolumn{5}{|l|}{ Matemática } \\
\hline Raciocínio Quantitativo & 13,11 & 3,02 & 5 & 18 \\
\hline Raciocínio Aditivo & 7,44 & 1,91 & 0 & 9 \\
\hline Raciocínio Multiplicativo & 5,16 & 2,36 & 0 & 9 \\
\hline
\end{tabular}

Fonte: elaborada pelas autoras. 


\section{Relações entre funções executivas e problemas de raciocínio quantitativo}

O desempenho matemático também apresentou relação com as funções executivas. Especificamente, tanto o total de acertos na tarefa de raciocínio quantitativo quanto o total de acertos separados por tipo de raciocínio (aditivo e multiplicativo) correlacionaramse com memória de trabalho ( $R Q$ : $r=0,372, p<0,01$; $R A: r=0,428, p<0,01$; $R M: r=0,269$, $p<0,05$ ) e fluência verbal (RQ: $r=0,424, p<0,01 ; R A: r=0,312, p<0,05 ; R M: r=0,288, p<0,05$ ). Isso significa dizer que quanto mais capacidade de memória de trabalho e de fluência verbal os estudantes tiveram, melhor foi o seu desempenho no raciocínio quantitativo. Tais resultados indicam que a resolução de problemas apresentados de forma oral é apoiada na memória de trabalho e na fluência verbal dos estudantes, o que é justificável ao levar em conta a necessidade de manter a informação na mente e manipulá-la para posteriormente resolver o problema. Esses resultados vão ao encontro de evidências da literatura que também apontam relações significativas entre memória de trabalho e resolução de problemas de raciocínio quantitativo (NOGUES; DORNELES, 2020), relações entre memória de trabalho e desempenho matemático geral (RAGHUBAR; BARNES; HECHT, 2010; HASSINGER-DAS et al., 2014) e o papel que a memória de trabalho desempenha nas dificuldades de aprendizagem em matemática (GATHERCOLE etal., 2016; TOLL et al., 2011).

\section{Relações entre compreensão de leitura e funções executivas}

A partir das análises estatísticas realizadas, foi possível verificar que, dentre as medidas de funções executivas, a atenção apresentou correlação significativa com todas as tarefas que avaliaram a leitura e a compreensão de leitura. Mais especificamente, a atenção correlacionou-se ${ }^{1}$ com todas as variáveis pertencentes ao Anele 1, ou seja, quanto mais capacidade atencional o estudante apresentou, melhor foi a sua leitura de palavras isoladas. Sobre o Anele 2, a atenção relacionou-se diretamente tanto com o total de acertos do questionário da tarefa $(r=0,310, p<0,05)$ quanto com o total de acertos do questionário inferencial $(r=0,381, p<0,01)$. No que se refere ao instrumento Anele 5 , a atenção apresentou-se correlacionada significativamente apenas com o tempo de leitura $(r=-0,280, p<0,05)$. A partir disso, pode-se inferir que a atenção é uma das funções executivas fundamentais para a leitura e compreensão, visto que os achados indicaram que quanto maior a capacidade de atenção do aluno, mais eficiente foi a sua leitura e a sua habilidade de compreender o texto.

Outra função executiva que pode ser destacada nessa relação é a memória de trabalho, a qual apresentou correlação positiva e significativa com os instrumentos Anele 1 e 2. Especificamente, no primeiro a memória de trabalho correlacionou-se com a leitura de palavras reais $(r=0,298, p<0,05)$ e com a leitura de palavras reais irregulares $(r=0,317, p<0,05)$. Sobre o segundo, as correlações significativas apareceram com o total de acertos do questionário $(r=0,443, p<0,01)$, detalhadamente com o total de acertos do questionário literal $(r=0,262, p<0,05)$ e do inferencial $(r=0,494, p<0,01)$, com o total de cláusulas $(r=0,427, p<0,01)$ e com a porcentagem da cadeia principal $(r=0,390$, $p<0,01)$, que se refere às partes centrais da história que o avaliado lembrou de recontar.

\footnotetext{
'Índices de correlação para cada variável: LPI total de acertos: $r=0,441, p<0,01$ : LPI Palavras reais: $r=0,379, p<0,01$; LPI Palavras Reais Regulares: $r=0,289, p<0,05$; LPI Palavras Reais Irregulares: $r=0,359, p<0,01$; LPI Pseudopalavras: $r=0,407$, $p<0,01$.
} 
Nesse sentido, é possível inferir que a memória de trabalho também está diretamente relacionada à habilidade de leitura e compreensão de leitura dos alunos, isto é, quanto maior a capacidade de memória de trabalho, melhor a leitura e a compreensão de leitura dos estudantes.

Além disso, cabe relatar que a variável fluência verbal, por sua vez, apresentou correlação negativa e significativa apenas com o tempo de leitura medido no Anele 5, indicando que quanto menor o tempo de leitura, melhor o desempenho na tarefa de fluência verbal e vice-versa. De outro modo, pode-se dizer que quanto maior a fluência verbal do estudante, mais rápida foi a sua leitura.

Os resultados reportados neste artigo, conforme o esperado, corroboram a literatura quanto às relações entre a leitura, em especial a compreensão, e as funções executivas (CORSO et al., 2016; JACOB; PARKINSON, 2015). Dentre os componentes das funções executivas, os achados são consistentes com a literatura ao apontar a memória de trabalho como uma habilidade cognitiva fundamental, tanto para a resolução de problemas quanto para a leitura e compreensão de leitura, conforme evidenciado pelas correlações positivas significativas tanto com as variáveis de matemática quanto com as de leitura e compreensão.

\section{Efeito de consistência}

A partir do teste de comparação não paramétrico de Wilcoxon verificou-se que o desempenho dos alunos nos problemas consistentes é significativamente melhor do que nos problemas inconsistentes $(Z=-5,46 ; p<0,001)$, como pode ser observado na Tabela 2.

Tabela 2 - Desempenho dos estudantes de acordo com a consistência dos problemas

\begin{tabular}{lccc}
\hline & Média (DP) & Mediana & Mínimo - Máximo \\
\hline Problemas Consistentes & $6,95(1,48)$ & 8,0 & $3,20-8,0$ \\
Problemas Inconsistentes & $5,36(1,57)$ & 5,0 & $0-8$ \\
\hline
\end{tabular}

Fonte: elaborada pelas autoras.

Esse resultado vai ao encontro da discussão proposta por Dowker e Nuerk (2017) sobre influências linguísticas no processamento numérico ou aritmético, no nível semântico. Nossos dados confirmam a existência do efeito de consistência: o nível semântico do enunciado, no caso deste estudo, lido oralmente, tem impacto na compreensão das instruções do problema. Questões com linguagem consistente com a operação requerida são mais fáceis do que aquelas com linguagem inconsistente, conforme evidenciado pela análise de comparação e sugerido no trabalho de Daroczy et al. (2017) e Dowker e Nuerk (2017).

\section{Considerações Finais}

De modo geral, as hipóteses estabelecidas neste estudo foram confirmadas. Fica clara a existência de relações importantes entre a compreensão de leitura, raciocínio quantitativo e funções executivas, além da presença do efeito de consistência nos problemas matemáticos. Os resultados deste estudo sugerem a necessidade de o 
trabalho escolar buscar integrar estas três dimensões, visando o favorecimento de aprendizagens significativas pelas crianças.

Pode-se concluir que as relações entre a tarefa de raciocínio quantitativo e as tarefas de reconhecimento de palavras e compreensão de leitura indicam a relevância da compreensão para resolução de problemas, com destaque para as inferências, não apenas quando o próprio aluno lê o enunciado, como também quando outra pessoa lê para ele. Este resultado destaca a necessidade de o trabalho pedagógico considerar a compreensão como essencial não só na área da leitura, como também na matemática: as ações colocadas em prática pelo estudante precisam ser compreendidas corretamente independentemente da área do conhecimento. Dessa forma, as relações evidenciadas neste estudo dão subsídios para abordagens que podem auxiliar a prática docente a possibilitar o favorecimento das aprendizagens dos alunos.

Além disso, foi evidenciado que tanto a tarefa de raciocínio quantitativo quanto as tarefas de leitura e compreensão leitora estão diretamente relacionadas com as funções executivas, com destaque para a memória de trabalho. Percebe-se, portanto, que este aspecto do funcionamento executivo é uma habilidade compartilhada entre o desempenho em leitura e em matemática, o que é um resultado importante ao se pensar no trabalho em sala de aula e nas tarefas a serem propostas aos estudantes, as quais podem incluir, também, tarefas de estímulo às funções executivas.

Considerando a verificação, entre os nossos dados, do efeito de consistência nos problemas de raciocínio quantitativo, pode-se compreender que o emprego do raciocínio quantitativo visando à resolução do problema é facilitado quando a linguagem utilizada é consistente com a operação requerida, enquanto os problemas inconsistentes impõem uma demanda inferencial maior. Entretanto, a implicação de tal resultado não significa priorizar problemas consistentes em detrimento dos inconsistentes. Pelo contrário, reconhecer a dificuldade imposta pela inconsistência entre as palavras e a operação a ser feita é uma oportunidade para investir em uma prática pedagógica que trabalhe com as diferenças da linguagem relacionadas à matemática, buscando, assim, favorecer o desenvolvimento não apenas do raciocínio quantitativo, como também da compreensão do aluno diante de problemas matemáticos que apresentam um enunciado de interpretação mais difícil.

Assim, acredita-se que um trabalho pedagógico baseado no reconhecimento das relações entre as áreas da leitura, da matemática e funções executivas seja promissor. Nesse sentido, os achados aqui discutidos dão suporte à prática em sala de aula, pois possibilitam a adoção de uma outra visão dos processos de ensino e de aprendizagem, principalmente considerando o papel das funções executivas tanto no desempenho em compreensão de leitura quanto em raciocínio quantitativo. Além disso, é válido ressaltar a leitura oral dos problemas matemáticos como uma alternativa possível diante de casos de alunos que apresentam dificuldades na leitura, sem esquecer que, independentemente da leitura do enunciado do problema ser realizada por ele ou pelo professor, a compreensão é fundamental para a resolução, assim como as funções executivas que apoiam esses processos. 


\section{Agradecimentos}

O presente trabalho foi realizado com apoio da Coordenação de Aperfeiçoamento de Pessoal de Nível Superior (Capes), Código de Financiamento 001.

This study was financed in part by the Brazilian Coordenação de Aperfeiçoamento de Pessoal de Nível Superior (CAPES), Financing Code 001.

\section{Referências}

ANGELINI, W.; ANGELINI, A.; ALVES, I. R. F. S.; CUSTODIO, E.; RAMÍREZ-DUARTE, W. F.; DUARTE, J. Matrizes progressivas coloridas de Raven: escala especial. São Paulo: CETEPP, 1999.

ASSOCIAÇÃO BRASILEIRA DE EMPRESAS E PESQUISA. Critério de classificação econômica Brasil: 2009. Disponível em: http://www.abep.org/criterio-brasil. Acesso em: 20 fev. 2019.

BASSO, F. P.; MINÁ, C. S.; PICCOLO, L. R.; SALLES, J. F. Coleção Anele 5: avaliação da fluência de leitura textual: AFLeT. São Paulo: Vetor, 2018.

BUSSAB, W. O.; MORETTIN, P. A. Estatística básica. 6. ed. São Paulo: Saraiva, 2010.

CIRINO, P. T.; FUCHS, L. S.; ELIAS, J.T.; POWELL, S. R.; SCHUMACHER, R. F. Cognitive and mathematical profiles for different forms of learning difficulties. Journal of Learning Disabilities, Chicago, v. 48, n. 2, p. 156-175, 2015. DOI: https://doi.org/10.1177/0022219413494239.

CONNOR, C. M. A lattice model of the development of reading comprehension. Child Developmental Perspectives, Washington, v. 10, n. 4, p. 269-274, 2016.

CORSO, H. V.; CROMLEY, J. G.; SPERB, T. M.; SALLES, J. F. Modeling the relationship among reading comprehension, intelligence, socioeconomic status and neuropsychological functions-the mediating role of executive functions. Psychology \& Neuroscience, Rio de Janeiro, v. 9, n. 1, p. 3245, 2016. DOI: https://doi.org/10.1037/pne0000036.

CORSO, H. V.; JOU, G. I.; FORNER, V. B.; PEREIRA, T. C. A. Treinamento do controle executivo no contexto da pesquisa e da clínica psicopedagógica. In: ROTTA, N. T.; BRIDI FILHO, C. A.; BRIDI, F. R. (org.). Plasticidade cerebral e aprendizagem: abordagem multidisciplinar. Porto Alegre: Artmed, 2018. p. 81-100.

CORSO, H. V.; PICCOLO, L. R.; MINÁ, C. S.; SALLES, J. F. Coleção Anele 2: avaliação da compreensão de leitura textual: comtext. São Paulo: Vetor, 2017.

CORSO, H. V.; SPERB, T. M.; SALLES, J. F. Compreensão leitora: modelos de processamento e relações com outras habilidades cognitivas. In: ROAZZI, A.; JUSTI, F. R. R.; SALLES, J. F. (org.). A aprendizagem da leitura e da escrita: contribuições de pesquisas. São Paulo: Vetor, 2013. p. 83108.

CORSO, L. V.; ASSIS, E. F. Reflexões acerca da aprendizagem inicial da matemática: contribuições de aspectos externos ao aluno. In: PICCOLI, L.; CORSO, L. V.; ANDRADE, S. S.; SPERRHAKE, R. (org.). Pacto nacional pela alfabetização na idade certa: PNAIC UFRGS: práticas de alfabetização, aprendizagem da matemática e políticas públicas. São Leopoldo: Oikos, 2018. p. 114-138. Disponível em: https://lume.ufrgs.br/handle/10183/186137. Acesso em: 27 jan. 2021.

CORSO, L. V.; DORNELES, B. V. Avaliação da matemática: competências numéricas e competências de base. In: SCICCHITANO, R. M. J.; CASTANHO, M. I. S. (org.). Avaliação psicopedagógica: recursos para a prática. Rio de Janeiro: Wak, 2013.

CORSO, L. V.; DORNELES, B. V. Perfil cognitivo dos alunos com dificuldades de aprendizagem na leitura e matemática. Psicologia: teoria e prática, São Paulo, v. 17, n. 2, p. 185-198, 2015. 
DAROCZY, G.; WOLSKA, M.; MEURERS, W. D.; NUERK, H.-C. Word problems: a review of linguistic and numerical factors contributing to their difficulty. In: DOWKER, A.; NUERK, H. Linguistic influences on mathematical cognition. Laussane: Frontiers Media, 2017. p. 22-34.

DIAMOND, A. Executive functions. Annual Review of Psychology, London, v. 64, p. 135-168, 2013.

DORNELES, B. V.; LIMA, E. M.; NOGUES, C. P. Aprendizagem da matemática nos anos iniciais: conceitos e desafios. In: PICCOLI, L.; CORSO, L. V.; ANDRADE, S. S.; SPERRHAKE, R. Pacto nacional pela alfabetização na idade certa: PNAIC UFRGS: práticas de alfabetização, aprendizagem da matemática e políticas públicas. São Leopoldo: Oikos, 2018. p. 139-158. Disponível em: https:// lume.ufrgs.br/handle/10183/186137. Acesso em: 27 jan. 2021.

DOWKER, A.; NUERK, H. Editorial: linguistic influences on mathematics. In: DOWKER, A.; NUERK, H. Linguistic influences on mathematical cognition. Laussane: Frontiers Media, 2017. p. 6-9.

FLETCHER, J. M.; LYONS, G. R.; FUCHS, L. S.; BARNES, M. A. Transtornos de aprendizagem: da identificação à intervenção. Porto Alegre: Artmed, 2009.

GATHERCOLE, S. E.; WOOLGAR, F.; KIEVIT, R. A.; ASTLE, D.; MANLY, T.; HOMES, J. How common are WM deficits in children with difficulties in reading and mathematics? Journal of Applied Research in Memory and Cognition, Amsterdam, v. 5, n. 4, p. 384-394, 2016.

HASSINGER-DAS, B.; JORDAN, N. C.; GLUTTING, J.; IRWIN, C.; DYSON, N. Domain-general mediators of the relation between kindergarten number sense and first-grade mathematics achievement. Journal of Experimental Child Psychology, New York, v. 118, p. 78-92, 2014. DOI: https://doi. org/10.1016/j.jecp.2013.09.008.

JACOB, R.; PARKINSON, J. The potential for school-based interventions that target executive function to improve academic achievement: a review. Review of Educational Research, Washington, v. 85, n. 4, p. 512-552, 2015.

KENDEOU, P.; VAN DEN BROEK, P.; HELDER, KARLSSON, J. A cognitive view of reading comprehension: implications for reading difficulties. Learning Disabilities: research \& practice, New York, v. 29, n. 1, p. 10-16, 2014. DOI: https://doi.org/10.1111/ldrp.12025.

MARTIN, R. B.; CIRINO, P. T.; SHARP, C.; BARNES, M. Number and counting skills in kindergarten as predictors of grade 1 mathematical skills. Learning and Individual Differences, Greenwich, USA, v. 34, p. 12-23, 2014. DOI: https://doi.org/10.1016/j.lindif.2014.05.006.

NOGUES, C. P.; DORNELES, B. V. Estimativa numérica, memória de trabalho e raciocínio quantitativo: relações no desempenho matemático. Zetetiké, Campinas, v. 28, e020022, p. 1-17, 2020. DOI: https://doi.org/10.20396/zet.v28i0.8655474.

NUNES, T. Teacher notes: family-school partnership to promote mathematics for deaf children. Oxford, UK: Oxford University, 2009. Disponível em: http://www.education.ox.ac.uk/ndcs/ Resources/teachersbook_exercises.pdf. Acesso em: 20 jan. 2019.

NUNES, T.; BRYANT, P.; BARROS, R.; SYLVA, K. The relative importance of two different mathematical abilities to mathematical achievement. British Journal of Educational Psychology, Edinburgh, v. 82, n. 1, p. 136-156, 2012. DOI: https://doi.org/10.1111/j.2044-8279.2011.02033.x

NUNES, T.; DORNELES, B. V.; LIN, P.-J.; RATHGEB-SCHNIERER, E. Teaching and learning about whole numbers in primary school. Cham: Springer, 2016. (ICME-13 Topical Surveys).

OECD. Programme for International Student Assessment (PISA): results from PISA 2018. [Paris]: OECD, 2019. Disponível em: https://www.oecd.org/pisa/publications/PISA2018_CN_BRA.pdf. Acesso em: 4 mar. 2020.

RAGHUBAR, K. P.; BARNES, M.; HECTH, S. Working memory and mathematics: a review of developmental, individual difference, and cognitive approaches. Learning and Individual Differences, Greenwich, USA, v. 20, n. 2, p. 110-122, 2010. 
SALLES, J. F.; PICCOLO, L. R.; MINÁ, C. S. Coleção Anele 1: avaliação de leitura de palavras e pseudopalavras isoladas: LPI. São Paulo: Vetor, 2017.

SALLES, J. F.; FONSECA, R. P.; PARENTE, M. A. M. P.; CRUZ-RODRIGUES, C.; MELLO, C. B.; BARBOSA, T.; MIRANDA, M. C. Instrumento de avaliação neuropsicológica breve infantil NEUPSILIN-INF. São Paulo: Vetor, 2016.

TOLL, S. W. M.; VAN DER VEN, S. H. G.; KROESBERGEN, E. H.; VAN LUIT, J. E. H. Executive functions as predictors of math learning disabilities. Journal of Learning Disabilities, Chicago, v. 44, n. 6, p. 521-532, 2011. DOI: https://doi.org/10.1177/0022219410387302.

VERSCHAFFEL, L; GREER, B; DE CORTE, E. Making sense of word problems. Lisse: Swets \& Zeitlinger, 2000. 